\title{
Análise da ansiedade e do estresse laboral em profissionais da Atenção Primária à
}

\section{Saúde}

\author{
Analysis of anxiety and work stress in primary health care professionals \\ Análisis de ansiedad y estrés laboral en profesionales de atención primaria
}

Pammela Weryka da Silva Santos

ORCID: https://orcid.org/0000-0002-9931-0951 Universidade Federal do Piauí, Brasil

E-mail: pammela_weryka@hotmail.com

Marisa Araujo Costa

ORCID: https://orcid.org/0000-0003-3711-6042

Universidade Estadual do Maranhão, Brasil

E-mail: marisa_mac81@ hotmail.com

Raydelane Grailea Silva Pinto

ORCID: https://orcid.org/0000-0001-8657-6462 Universidade Federal do Piauí, Brasil

E-mail: raydelane.silva@gmail.com

Kameny Santos Franco

ORCID: https://orcid.org/0000-0001-6056-3663

Centro Universitário Santo Agostinho, Brasil

E-mail: kamenyfranco@gmail.com

Raquel Ferreira Coelho

ORCID: https://orcid.org/0000-0003-2988-544X

Universidade Federal do Piauí, Brasil

E-mail: raquelxcoelho@hotmail.com

Yasmine Maria Rodrigues dos Santos

ORCID: https://orcid.org/0000-0001-5519-4795

Centro Universitário Santo Agostinho, Brasil yasminemine2009@ hotmail.com

Ana Hélia de Lima Sardinha

ORCID: https://orcid.org/0000-0002-8720-6348

Universidade Federal do Maranhão, Brasil

E-mail: ana.helia@ufma.br

\section{Resumo}

Objetivo: Analisar o nível de ansiedade e estresse laboral em trabalhadores das Unidades Básica de Saúde de CaxiasMA. Metodologia: Trata-se de um estudo analítico, de corte transversal e abordagem quantitativa. Setenta e um (71) trabalhadores responderam o questionário sociodemográfico e ocupacional, a escala de ansiedade, Inventário de Ansiedade Traço-Estado (IDATE) e estresse, Escala de Estresse no Trabalho (EET). Os dados provenientes dos instrumentos metodológicos foram tabulados através do programa Office Excel versão 2013, sendo criado um banco de dados, e posteriormente, analisados eletronicamente pelo programa SPSS 22.0 para o Windows. Resultados e discussão: Evidenciou-se a predominância do nível médio de ansiedade e de fatores estressantes, sendo os vigilantes os mais ansiosos e estressados. Trabalhadores com mais de uma ocupação apresentam maiores níveis de ansiedade. Sobre o Distúrbio neuromusculoesquelético (DNME), verifica-se a predominância entre os vigias, porém em todas as profissões presenciou-se a existência deste distúrbio, exceto recepcionista. Os resultados apontam que dos 21 trabalhadores com outra ocupação, 11 possuem DNME. Conclusão: Torna-se necessário desenvolver um olhar mais cuidadoso para a saúde física e mental dos trabalhadores como o fortalecimento do apoio social no trabalho, a importância da ergonomia, dos momentos de interação em equipe, escuta qualificada, além de atividades que possam enriquecer e reorganizar o processo e o fluxo de trabalho, junto com o assessoramento da gestão e dos órgãos da vigilância. Mediante limitações da pesquisa é de grande importância o surgimento de outros estudos sobre o cuidado com a saúde do trabalhador.

Palavras-chave: Saúde do trabalhador; Ansiedade; Estresse; Estresse ocupacional; Atenção primária à saúde.

\begin{abstract}
Objective: To analyze the level of anxiety and work stress in workers of the Basic Health Units of Caxias-MA. Methodology: This is an analytical, cross-sectional study with a quantitative approach. Seventy-one (71) workers answered the sociodemographic and occupational questionnaire, the anxiety scales, Trait-State Anxiety Inventory (STAI) and stress, Stress at Work Scale (TSE). The data from the methodological instruments were tabulated using the Office Excel version 2013 program, a database was created, and later, analyzed electronically by the SPSS 22.0
\end{abstract}


program for Windows. Results and discussion: The predominance of the average level of anxiety and stressful factors was evidenced, with the vigilant ones being the most anxious and stressed. Workers with more than one occupation have higher levels of anxiety. Regarding Neuromusculoskeletal Disorder (DNME), there is a predominance among the watchmen, however in all professions the existence of this disorder was observed, except receptionist. The results show that of the 21 workers with another occupation, 11 have DNME. Conclusion: It is necessary to develop a more careful look at the physical and mental health of workers such as strengthening social support at work, the importance of ergonomics, moments of team interaction, qualified listening, in addition to activities that can enrich and reorganize the process and workflow, together with the advice of management and supervisory bodies. Due to research limitations, the emergence of other studies on care for workers' health is of great importance.

Keywords: Worker's health; Anxiety; Stress; Occupational stress; Primary health care.

\section{Resumen}

Objetivo: Analizar el nivel de ansiedad y estrés laboral en trabajadores de las Unidades Básicas de Salud de CaxiasMA. Metodología: Se trata de un estudio analítico, transversal con enfoque cuantitativo. Setenta y un (71) trabajadores respondieron el cuestionario sociodemográfico y ocupacional, las escalas de ansiedad, el Inventario Rasgo-Estado de Ansiedad (STAI) y el Estrés, la Escala de Estrés en el Trabajo (TSE). Los datos de los instrumentos metodológicos se tabularon utilizando el programa Office Excel versión 2013, se creó una base de datos y posteriormente se analizó electrónicamente con el programa SPSS 22.0 para Windows. Resultados y discusión: Se evidenció el predominio del nivel promedio de ansiedad y estresores, siendo los vigilantes los más ansiosos y estresados. Los trabajadores con más de una ocupación tienen niveles más altos de ansiedad. En cuanto al Trastorno Neuromusculoesquelético (DNME), existe un predominio entre los vigilantes, sin embargo en todas las profesiones se observó la existencia de este trastorno, excepto el de recepcionista. Los resultados muestran que de los 21 trabajadores con otra ocupación, 11 tienen DNME. Conclusión: Se hace necesario desarrollar una mirada más atenta a la salud física y mental de los trabajadores, como el fortalecimiento del apoyo social en el trabajo, la importancia de la ergonomía, momentos de interacción en equipo, escucha calificada, además de actividades que puedan enriquecer y reorganizar. el proceso y flujo de trabajo, junto con el asesoramiento de los órganos de dirección y supervisión. Debido a las limitaciones de la investigación, la aparición de otros estudios sobre el cuidado de la salud de los trabajadores es de gran importancia.

Palabras clave: Salud del trabajador; Ansiedad; Estrés; Estrés ocupacional; Atención primaria.

\section{Introdução}

No Brasil, a Saúde do Trabalhador inicia seu processo de organização desde os anos 1980, mais precisamente a partir da Reforma Sanitária. O Sistema Único de Saúde (SUS) estabelece uma relação de responsabilidade com todo indivíduo e com sua vida laboral ativa (Brasil, 2018).

A ocupação representa um dos fatores determinantes da situação de saúde dos trabalhadores, podendo colaborar para fortalecê-la ou desgastá-la. Os sujeitos gastam maior parte do tempo de suas vidas dedicado à atividade laboral, seja dentro ou fora do ambiente e expediente de trabalho. Desta forma, o trabalho deveria significar realização, satisfação e prazer. Porém, de forma especial na sociedade capitalista, a ocupação se apresenta como um meio de exploração e desigualdade social, e, consequentemente, atua como fonte ou intensificador de problemas de saúde (Carneiro, Peixoto \& Albuquerque, 2021).

A atenção com o trabalhador vem ganhando espaço, uma vez que as jornadas de trabalho estão cada vez mais excessivas e o lazer mais escasso, afetando negativamente na saúde física e mental do trabalhador (Laux, Hoff, Antes, Cviatkovski \& Corazza, 2018). Nota-se uma forte resistência social em reconhecer que o trabalho pode ter consequências como, mal-estar, doenças e acidentes durante o exercício da profissão. Tal resistência, deve-se a diversos interesses e poderes envolvidos e tem levado a sociedade a esconder o adoecimento no trabalho (Cardoso \& Morgado, 2019).

Transformações nas condições de saúde dos profissionais podem levar à relevante redução da capacidade ou à incapacidade para o trabalho, e como consequência à perda ou diminuição de produtividade, seja pelo absenteísmo ou presenteísmo. Define-se como presenteísmo estar no trabalho mesmo percebendo limitações, físicas ou mentais, que podem reduzir a capacidade laboral (Pie, Fernandes, Carvalho \& Porto, 2020).

O estudo de Carlotto, Câmara, Batista \& Schneider (2019) evidencia que a realidade na qual o trabalhador está inserido é responsável pelo aumento do número de adoecimentos relacionados a atividade laboral, uma vez constatada a 
presença de disfunções osteomusculares e desequilíbrios psíquicos, surgindo assim os transtornos mentais como a ansiedade e o estado de estresse.

O estresse ocupacional contínuo pode acarretar em prejuízos à saúde mental e física do trabalhador, tais como: hipertensão, diabetes, o desenvolvimento da síndrome metabólica, distúrbios do sono, enfermidades psicossomáticas, síndrome de Burnout, depressão, uso de substâncias psicoativas, além de queda na produtividade, absenteísmo, insatisfação laboral e baixa qualidade de vida no trabalho (Ribeiro et al., 2015).

No que se refere à ansiedade, Brentini, Brentini, Araújo, Aros \& Aros (2018), ao realizar uma revisão sobre o transtorno de ansiedade generalizada no contexto clínico e social no âmbito da saúde mental, identifica que este distúrbio é o mais prevalente dentre os transtornos mentais (prevalência de 12,6\%/ano), demonstrando a necessidade de desenvolver estratégias para reverter esta prevalência.

Dentre as doenças relacionadas com a saúde do trabalhador, os distúrbios osteomusculares relacionados ao trabalho (DORT) são os mais presentes, gerando desconforto e dores em diferentes graduações de intensidades, além de comprometer vários segmentos corporais. Assim, as condições de trabalho insalubres influenciam no aparecimento dos distúrbios neuromusculoesqueléticos (DNME) favorecendo o adoecimento mental dos trabalhadores, principalmente o estresse e ansiedade laboral, repercutindo negativamente na qualidade de vida dos profissionais, consequentemente no aspecto financeiro das empresas (Dosea, Oliveira \& Lima, 2016).

Mediante exposto por Fernandes et al. (2018), os profissionais da área de saúde rotineiramente se deparam com situações complexas que podem acarretar consequências negativas para sua condição biopsicossocial. Na Atenção Primária à Saúde (APS), a jornada de trabalho consiste em uma rotina na qual a equipe está diariamente exposta a fatores que podem prejudicar seu corpo e mente, como o espaço físico ergonomicamente incorreto, fragilidade de recursos humanos e materiais, cobrança exacerbada pela população assistida, conflitos pessoais e interdisciplinares entre a equipe, e apoio deficiente pela gestão.

Deste modo, com o intuito de analisar a presença de ansiedade e estresse de trabalhadores da APS, a pesquisa foi desenvolvida em quatro Unidades Básicas de Saúde (UBS) do município de Caxias/MA, uma vez que não há relatos na literatura sobre estudos voltados para a saúde destes trabalhadores em questão, justificando a realização deste estudo.

O objetivo do estudo foi analisar o nível de ansiedade e estresse laboral entre trabalhadores das Unidades Básica de Saúde de Caxias - MA.

\section{Metodologia}

\section{Tipo de estudo}

Trata-se de um estudo analítico, de corte transversal e abordagem quantitativa.

\section{Local do estudo}

A pesquisa foi realizada na UBS da Baixinha, do Luiza Queiroz, do Piquizeiro e da Trezidela, todas localizadas em Caxias, Maranhão, Brasil.

Estes estabelecimentos de saúde contam com o apoio de uma equipe do Núcleo Ampliado de Saúde da Família (NASF). Fornecem serviços de acolhimento com classificação de risco, estratificação de risco familiar, assistência multiprofissional, consultas com médico, enfermeiros e dentista, ações de Vigilância em Saúde, dentre outros serviços. 


\section{População do estudo}

A população foi composta por 82 trabalhadores, sendo 20 profissionais da UBS da Baixinha, 14 da UBS Luiza Queiroz, 25 da UBS Piquizeiro e 23 trabalhadores da UBS da Trezidela, incluindo médicos, enfermeiros, dentistas, auxiliares de saúde bucal (ASB), agentes comunitários de saúde (ACS), técnicos de enfermagem, recepcionistas, agentes de serviços gerais (AOSD) e vigias. Todas as unidades são compostas por uma Equipe de Saúde da Família (ESF), com exceção da UBS Piquizeiro que é formada por duas equipes.

Entretanto, apenas 71 fizeram parte da pesquisa, pois 04 profissionais estavam de férias, 02 de licença médica, e 05 se recusaram a participar.

\section{Critérios de inclusão}

Profissionais que trabalham ativamente há pelo menos um ano na APS, de Caxias/MA.

\section{Critérios de exclusão}

Profissionais que estiverem de férias, de licença de qualquer natureza, e/ou afastados com atestado médico, ou pelo Instituto Nacional do Seguro Social (INSS).

\section{Instrumentos de coleta de dados}

- Questionário sociodemográfico e ocupacional

O questionário foi elaborado pelas pesquisadoras do estudo, é constituído por 06 questões referentes ao perfil sociodemográfico que investiga sexo, idade, escolaridade, quantidade de filhos e o grau de responsabilidade do trabalhador na manutenção financeira de sua família, seguido de 09 indagações sobre o perfil ocupacional dos mesmos.

- Inventário de Ansiedade Traço-Estado (IDATE)

O Inventário de Ansiedade Traço-Estado trata-se de um instrumento validado desde 1970, referenciado com frequência na literatura, e utilizado tanto na clínica como em estudos científicos. Foi embasado na teoria Ansiedade traçoestado de Spielberger (1970), que consiste em afirmar que o tipo traço se configura por ser uma condição emocional transitória mediante exposição aos diversos estímulos. Por outro lado, o estado é característico por ser uma reação comportamental, por sua vez estável, e varia entre os indivíduos, caracterizando assim a pessoa em hipersensível, ou seja, bastante responsivo psicologicamente. Este inventário era utilizado na versão STAI (State-Trait Personality Inventory), e posteriormente, foi traduzido e validado para o português por Biaggio (1980), sendo reconhecido como IDATE, no Brasil (Kaipper, 2008).

Este instrumento avalia a Ansiedade Traço-Estado separadamente, enquanto na classificação Estado se investiga como o funcionário tem se sentido hoje, na outra averigua como geralmente ele se sente. Deste modo, para cada categoria a ferramenta fornece 20 questões, com múltiplas escolhas (1- Absolutamente não; 2- Um pouco; 3- Bastante; 4- Muitíssimo). Cada resposta possui uma pontuação específica, sendo atribuída uma pontuação final através da somatória de todos os itens, gerando assim uma interpretação em baixo, médio, ou alto nível de ansiedade.

- Escala de Estresse no Trabalho (EET)

A Escala de Estresse no Trabalho refere-se a uma escala que avalia o estado de estresse no espaço laboral, proposta e validada desde 2004, por Paschoal e Tamayo. Sua estrutura é composta por 23 afirmativas baseadas nos estressores psicossociais e nas reações psicológicas ao estresse laboral. Cada item possibilita ao trabalhador pontuar o quanto concorda ou não com o quesito, uma vez que contempla as respostas (1- Discordo totalmente; 2- Discordo; 3- Concordo em parte; 4Concordo; 5- Concordo totalmente), logo quanto menor o número da resposta, maior a discordância da afirmativa, pelo entrevistado (Almeida, 2015). 


\section{Procedimento de coleta de dados}

Em reunião com as equipes, em seus respectivos locais de trabalho, foi explicado sobre o projeto, logo depois solicitou que os mesmos assinassem o Termo de Consentimento Livre e Esclarecido (TCLE), este sendo duas vias, mantendo uma em seu poder e a outra com a pesquisadora. Posteriormente, de acordo com a disponibilidade de cada trabalhador, em uma sala do próprio estabelecimento de saúde, foram aplicados individualmente os instrumentos metodológicos (questionário sociodemográfico e as escalas de ansiedade e de estresse), e caso surgisse alguma dificuldade durante o preenchimento, ofertou-se todo auxílio necessário.

Em outro momento foi realizado uma ação de educação em saúde com palestra e dinâmicas, como ginástica laboral e exercício de relaxamento, cujo objetivo era explanar sobre a saúde do trabalhador e repercussões no sistema musculoesquelético e na saúde mental, assim como esclarecer possíveis dúvidas.

\section{Análise estatística}

Os dados provenientes dos instrumentos metodológicos foram tabulados através do programa Office Excel versão 2013, sendo criado um banco de dados, e posteriormente, analisados eletronicamente pelo programa SPSS 22.0 para o Windows. Assim desenvolveu-se uma análise estatística descritiva, realizando o cálculo da média e do desvio padrão para examinar as varáveis numéricas. As variáveis categóricas foram representadas por meio de frequências absolutas e relativas. Para verificar a normalidade dos dados utilizou-se o Teste Shapiro Wilk.

Para estabelecer possíveis associações entre estresse e ansiedade (variáveis dependentes) com as características sociodemográficas e profissionais dos participantes foram empregados os Testes: Teste Qui-quadrado de Pearson, nos casos de dados com distribuição normal, ou Teste Exato de Fisher e Teste de Kruskal-Wallis, no caso dos dados com distribuição não paramétrica. Para todas as análises serão consideradas estatisticamente significantes valores de $p \leq 0,05$.

\section{Aspectos Ético-legais}

O projeto de pesquisa foi submetido à Plataforma Brasil e, em seguida, direcionado ao Comitê de Ética em Pesquisa, $\mathrm{n}^{\circ}$ 3.692.397, da Universidade Estadual do Maranhão (UEMA) e CAAE 22344719.5.0000.5554. As pesquisadoras, responsáveis e participantes seguiram as normas preconizadas pela Resolução do CNS 466/12 e suas complementares.

Após a autorização dos estabelecimentos de saúde onde foi feito o estudo, iniciou a coleta de dados mediante assinatura do TCLE por parte das participantes, assegurando as condições legais necessárias.

\section{Resultados e Discussão}

A tabela 1 mostra o perfil sociodemográfico dos entrevistados. Observa-se que 71,8\% dos participantes são do sexo feminino e com idade média de aproximadamente 42 anos. Sendo que 37,3\% dos entrevistados possuem ensino superior e 47,1\% casado (a) / União Estável. 
Tabela 1- Características sociodemográficas dos profissionais.

\begin{tabular}{|l|c|c|}
\hline \multicolumn{1}{|c|}{ Variável } & N & $\%$ \\
\hline Sexo & & 71 \\
\hline Feminino & 20 & 28,2 \\
\hline Masculino & & 1,8 \\
\hline Faixa etária & 10 & 14,1 \\
\hline Até 30 anos & 27 & 38 \\
\hline De 31 a 40 anos & 16 & 22,5 \\
\hline De 41 a 50 anos & 18 & 25,4 \\
\hline Acima de 50 anos & & \\
\hline Idade & & 41,8 \\
\hline Média \pm Desvio-padrão & $\pm 11,2$ \\
\hline Estado Civil & 27 & \\
\hline Solteiro (a) & 33 & 38,6 \\
\hline Casado (a)/ União Estável & 8 & 47,1 \\
\hline Separado (a) & 2 & 11,4 \\
\hline Viúvo (a) & & 2,9 \\
\hline Escolaridade & 9 & 13,4 \\
\hline Ens. Fundamental & 20 & 29,9 \\
\hline Ens. Médio & 25 & 37,3 \\
\hline Ens. Superior & 13 & 19,4 \\
\hline Pós-Graduação & & \\
\hline
\end{tabular}

Fonte: Autores (2020).

Na tabela 2 é possível observar as características sociais e de renda dos entrevistados. Temos que 42,3\% dos respondentes afirmam dividir igualmente as responsabilidades financeiras. Verifica-se também que 51,5\% estão com mais de 10 anos no trabalho atual e 70,4\% não possuem outra ocupação. O tempo médio de diagnóstico médico do DNME analisado foi de 9,8 anos. Concluindo assim uma amostra com características sociodemográficas bem diversificadas.

Tabela 2- Características sociais dos profissionais.

\begin{tabular}{|c|c|c|}
\hline \multirow{2}{*}{\multicolumn{3}{|c|}{ Responsabilidade Financeira }} \\
\hline & & \\
\hline Único responsável & 25 & 35,2 \\
\hline Principal responsável & 8 & 11,3 \\
\hline Contribui apenas & 5 & 7 \\
\hline Divide igual & 30 & 42,3 \\
\hline Não tem & 3 & 4,2 \\
\hline \multicolumn{3}{|l|}{ Profissão } \\
\hline AOSD/ASB/ACS & 35 & 49,3 \\
\hline Médico (a)/ Enfermeiro (a) / Téc. Enfermagem & 19 & 26,8 \\
\hline Dentista & 4 & 5,6 \\
\hline Recepcionista & 2 & 2,8 \\
\hline Vigia & 11 & 15,5 \\
\hline \multicolumn{3}{|l|}{ Tempo no atual emprego } \\
\hline Até 1 ano & 11 & 16,2 \\
\hline De 1 ano a 10 anos & 22 & 32,4 \\
\hline Acima de 10 anos & 35 & 51,5 \\
\hline \multicolumn{3}{|l|}{ Outra ocupação } \\
\hline Sim & 21 & 29,6 \\
\hline Não & 50 & 70,4 \\
\hline \multicolumn{3}{|l|}{ DNME } \\
\hline Sim & 26 & 36,6 \\
\hline Não & 45 & 63,4 \\
\hline \multicolumn{3}{|l|}{ Tempo de Diagnóstico } \\
\hline Média \pm Desvio-padrão & \multicolumn{2}{|c|}{$9,8 \pm 8,6$} \\
\hline
\end{tabular}

Fonte: Autores (2020). 
O âmbito da saúde exige um grupo de trabalhadores heterogêneos com características, especificidades e relações distintas que vão desde as diferentes formas de organização, contratação, até os processos de produção. Deste modo, este universo é numeroso e diversificado, abrangendo competências, habilidades, especialidades, cargos, funções, idades, rendas, escolaridades e diferentes níveis de autonomia no desempenho do processo de trabalho (Carlos, 2017).

Tabela 3- Escala de Ansiedade dos profissionais.

\begin{tabular}{|c|c|c|}
\hline Escala de Ansiedade & $\mathbf{N}$ & $\%$ \\
\hline \multicolumn{3}{|l|}{ Estado } \\
\hline Baixo & 23 & 32,4 \\
\hline Médio & 48 & 67,6 \\
\hline \multicolumn{3}{|l|}{ Traço } \\
\hline Baixo & 27 & 38,0 \\
\hline Médio & 40 & 56,3 \\
\hline Alto & 4 & 5,7 \\
\hline
\end{tabular}

Fonte: Autores (2020).

Como resultado da escala de ansiedade (estado e traço), observa-se na tabela 3, que em relação ao estado, 67,6\% apresentam ansiedade no nível médio e 32,4\% no nível baixo. Já no que diz respeito ao traço, observamos que a maioria, aproximadamente 56,3\% apresentam nível médio, 38\% nível baixo e apenas 5,7\% com nível alto.

O estresse e a ansiedade são comuns na área da saúde. O trabalho em ambientes (unidades de saúde, hospitais, ambulatórios e similares) impacta não somente no registro de acidentes de trabalho, mas também no desencadeamento de frequentes situações de estresse, ansiedade e de fadiga emocional. Nestes ambientes existem diversas fontes geradoras de ansiedade e estresse como a carência de concursos públicos, recursos físicos e materiais insuficientes, contratos precários, baixa valorização do trabalho pelos usuários, as relações de trabalho, as próprias atividades laborais por demandarem ações coletivas, uso das tecnologias leves, entre outras (Carlos, 2017).

A ansiedade pode ser classificada como AE ou AT. A primeira trata-se de uma ansiedade momentânea, ou seja, caracteriza a ansiedade do indivíduo em um dado momento. A segunda tem relação com a personalidade, logo investiga como geralmente o trabalhador considera seu nível de ansiedade. Nota-se que a AE pode variar em intensidade, podendo apresentar nível alto em um determinado momento e em outro não, enquanto a AT é caracterizada por ser mais estável (Fernandes, Chaves Neto, Trajano \& Braga, 2018b). Deste modo, durante a aplicação das escalas de ansiedade os trabalhadores referem não estarem ansiosos, logo nível baixo de AE. Por outro lado, ao serem questionados sobre a AT, os mesmos afirmam que constantemente sente-se altamente ansioso. Tal fato justifica a presença do nível alto na AT quando comparado com a AE, na qual apresentou apenas os níveis baixo e médio. 
Tabela 4- Média das respostas da Escala de Estresse no Trabalho - EET.

\begin{tabular}{|c|c|c|c|}
\hline Fator & Item & Média & Desvio-padrão \\
\hline Fator 1 & $\begin{array}{l}\text { A forma como as tarefas são distribuídas em minha área tem me deixado } \\
\text { nervoso }\end{array}$ & 2,4 & 1,1 \\
\hline Fator 2 & O tipo de controle existente em meu trabalho me irrita & 2,0 & 0,9 \\
\hline Fator 3 & A falta de autonomia na execução do meu trabalho tem sido desgastante & 1,9 & 0,9 \\
\hline Fator 4 & $\begin{array}{l}\text { Tenho me sentido incomodado com a falta de confiança de meu superior } \\
\text { sobre meu trabalho }\end{array}$ & 1,5 & 0,8 \\
\hline Fator 5 & $\begin{array}{l}\text { Sinto-me irritado com a deficiência na divulgação de informações sobre } \\
\text { decisões organizacionais }\end{array}$ & 2,1 & 0,9 \\
\hline Fator 6 & $\begin{array}{l}\text { Sinto-me incomodado com a falta de informações sobre minhas tarefas no } \\
\text { trabalho }\end{array}$ & 1,9 & 0,9 \\
\hline Fator 7 & $\begin{array}{l}\text { A falta de comunicação entre mim e meus colegas de trabalho deixa-me } \\
\text { irritado }\end{array}$ & 1,9 & 1,1 \\
\hline Fator 8 & $\begin{array}{l}\text { Sinto-me incomodado por meu superior tratar-me mal na frente de colegas } \\
\text { de trabalho }\end{array}$ & 1,5 & 1,0 \\
\hline Fator 9 & $\begin{array}{l}\text { Sinto-me incomodado por ter que realizar tarefas que estão além de minha } \\
\text { capacidade }\end{array}$ & 2,1 & 1,2 \\
\hline Fator 10 & Fico de mau humor por ter que trabalhar durante muitas horas seguidas & 1,8 & 1,0 \\
\hline Fator 11 & $\begin{array}{l}\text { Sinto-me incomodado com a comunicação existente entre mim e meu } \\
\text { superior }\end{array}$ & 1,5 & 0,7 \\
\hline Fator 12 & Fico irritado com discriminação/favoritismo no meu ambiente de trabalho & 1,9 & 0,9 \\
\hline Fator 13 & $\begin{array}{l}\text { Tenho me sentido incomodado com a deficiência nos treinamentos para } \\
\text { capacitação profissional }\end{array}$ & 2,3 & 1,3 \\
\hline Fator 14 & Fico de mau humor por me sentir isolado na organização & 1,7 & 0,8 \\
\hline Fator 15 & Fico irritado por ser pouco valorizado por meus superiores & 1,8 & 1,0 \\
\hline Fator 16 & $\begin{array}{l}\text { As poucas perspectivas de crescimento na carreira tem me deixado } \\
\text { angustiado }\end{array}$ & 2,3 & 1,2 \\
\hline Fator 17 & $\begin{array}{l}\text { Tenho me sentido incomodado por trabalhar em tarefas abaixo do meu } \\
\text { nível de habilidade }\end{array}$ & 1,8 & 0,8 \\
\hline Fator 18 & $\begin{array}{l}\text { A competição no meu ambiente de trabalho tem me deixado de mau } \\
\text { humor }\end{array}$ & 1,6 & 0,9 \\
\hline Fator 19 & $\begin{array}{l}\text { A falta de compreensão sobre quais são minhas responsabilidades neste } \\
\text { trabalho tem causado irritação }\end{array}$ & 1,7 & 0,8 \\
\hline Fator 20 & Tenho estado nervoso por meu superior me dar ordens contraditórias & 1,6 & 0,9 \\
\hline Fator 21 & $\begin{array}{l}\text { Sinto-me irritado por meu superior encobrir meu trabalho bem feito diante } \\
\text { de outras pessoas }\end{array}$ & 1,7 & 0,9 \\
\hline Fator 22 & $\begin{array}{l}\text { O tempo insuficiente para realizar meu volume de trabalho deixa-me } \\
\text { nervoso }\end{array}$ & 2,1 & 1,1 \\
\hline Fator 23 & $\begin{array}{l}\text { Fico incomodado por meu superior evitar me incumbir de } \\
\text { responsabilidades importantes }\end{array}$ & 1,7 & 0,9 \\
\hline
\end{tabular}

Fonte: Autores (2020).

Ao analisar a tabela 4, notam-se as médias e os desvios-padrões das respostas dos fatores da EET. Observa-se que os fatores estressores que obtiveram médias mais altas referem-se à deficiência nos treinamentos para capacitação profissional (fator 13: 2,3), as poucas perspectivas de crescimento na carreira (fator 16: 2,3) e a forma como as tarefas são distribuídas (fator $1: 2,4)$.

Em concordância com nossos achados, o estudo de Carlos (2017) que teve como objetivo traçar o perfil socioprofissional, analisar a prevalência do estresse no trabalho, a qualidade de vida e o grau de risco do uso e abuso de álcool dos trabalhadores da ESF, evidenciou ao aplicar a EET, que os fatores mais estressantes foram à deficiência nos treinamentos para capacitação profissional, as poucas perspectivas de crescimento na carreira, a forma como as tarefas são distribuídas, o tempo insuficiente para realizar o trabalho, divulgação de informações sobre decisões organizacionais e realização de tarefas que estão além da capacidade. 
O fator 1 refere-se a forma como as tarefas são distribuídas, ou seja, característica oriunda do processo de organização do trabalho. Sabe-se que a demanda de trabalho na Saúde da Família engloba desde a promoção da saúde até o tratamento e reabilitação, exigindo dos profissionais uma dinâmica de serviço integrada, participativa e resolutiva, caracterizando este universo laboral como específico e complexo (Brasil, 2012). Neste contexto, são exigidos do profissional requisitos como empoderamento, habilidades, competências, formação, qualificação que não se restringem aos conhecimentos da graduação, e sim a uma possível pós-graduação em Saúde da Família (Oliveira, 2007).

Alguns questionamentos podem ser levantados para justificar esta insatisfação sobre a distribuição das tarefas como, se a especialidade destes profissionais está voltada para o eixo de Saúde da Família, pois neste estudo não foi investigado se essa pós-graduação foi na área de saúde da família/saúde pública/saúde coletiva, uma vez que apenas 19,4\% dos participantes possuem pós-graduação. Além de investigar como estar à execução da educação permanente dentro das unidades e se existe um apoio da gestão mediante este cenário, uma vez que a falta de treinamentos e capacitação profissional foi outro fator elencado pelos entrevistados.

Segundo Carlos (2017) a capacitação profissional enriquece o desenvolvimento das atividades laborais, uma vez que acredita no potencial do profissional e lhe proporciona um enriquecimento intelectual. O estímulo à capacitação é um dos direcionamentos da atenção básica, sendo de responsabilidade das três esferas governamentais. Cabe aos gestores identificar as necessidades de seus profissionais e estimular a sua capacitação, oferecendo cursos e buscando apoiadores como as universidades (Souza \& Stancato, 2010). Outro fator estressante levantado baseia-se nas poucas perspectivas de crescimento na carreira dentro da instituição, na qual pode estar associado aos vínculos empregatícios prevalentes como celetistas, terceirizados, com predominância do vínculo por contrato anual.

Ascenção na carreira profissional enriquece a motivação para trabalhar, e quando isso não acontece impacta negativamente na vida pessoal, profissional, social, e na qualidade de vida dos profissionais. Sonhos de salários melhores, reconhecimento por meio de promoções, condição financeira para adesão de imóveis e automóveis, são motivadores para os trabalhadores, porém não isenta a importância da determinação e esforço pessoal, foco, e planejamento para alcançar suas metas e sonhos (Morin, Tonelli \& Pliopas, 2007).

Tabela 5- Associação entre os níveis de ansiedade e estresse por profissão.

\begin{tabular}{|c|c|c|c|c|c|c|}
\hline \multirow[t]{2}{*}{ Ansiedade } & \multicolumn{5}{|c|}{ Profissão } & \multirow[t]{2}{*}{ p-valor } \\
\hline & AOSD/ASB/ACS & $\begin{array}{c}\text { Médico (a) / } \\
\text { Enfermeiro } \\
\text { (a) / Téc. } \\
\text { Enfermagem }\end{array}$ & Dentista & Recepcionista & Vigia & \\
\hline \multicolumn{7}{|l|}{ Estado } \\
\hline Baixo & $40,0(14)$ & $26,3(5)$ & $25,0(1)$ & $50,0(1)$ & $18,2(2)$ & \multirow[t]{2}{*}{$0,619^{Q}$} \\
\hline Médio & $60,0(21)$ & $73,7(4)$ & $75,0(3)$ & $50,0(1)$ & $81,8(9)$ & \\
\hline \multicolumn{7}{|l|}{ Traço } \\
\hline Baixo & $37,1(13)$ & $52,6(10)$ & $50,0(2)$ & $0,0(0)$ & $18,2(2)$ & \multirow[t]{3}{*}{$0,131^{\mathbf{Q}}$} \\
\hline Médio & $57,1(20)$ & $47,4(9)$ & $50,0(2)$ & $50,0(1)$ & $72,7(8)$ & \\
\hline Alto & $5,7(2)$ & $0,0(0)$ & $0,0(0)$ & $50,0(1)$ & $9,1(1)$ & \\
\hline \multicolumn{7}{|l|}{ Estresse no Trabalho } \\
\hline Média \pm Desvio-padrão & $1,8 \pm 0,53$ & $1,7 \pm 0,74$ & $1,8 \pm 0,48$ & $1,6 \pm 0,86$ & $2,2 \pm 0,54$ & $0,345^{\mathrm{K}}$ \\
\hline
\end{tabular}

Q Teste Qui-Quadrado (nível de $95 \%$ de confiança). ${ }^{\mathbf{K}}$ Teste de Kruskal-Wallis (nível de $95 \%$ de confiança). Fonte: Autores (2020).

Para verificar se existe associação entre a profissão e os níveis de ansiedade e estresse nos participantes, aplicamos o teste Qui-Quadrado e o teste de Kruskal-Wallis, ambos ao nível de 95\% de confiança. Pelos resultados obtidos na Tabela 5 acima, não houve associação significativa entre as variáveis (p-valor > 0,05). Um dos motivos para tal resultado deve ser 
pautado em que cada categoria profissional tem um processo de trabalho específico na qual requer demandas psicológicas, físicas e emocionais distintas, que devem ser analisadas com mais cautela (Almeida, Ribeiro, Montibeler, Domingues Junior \& Saldanha, 2017). No entanto o objetivo deste estudo não está voltado para uma análise mais minuciosa desta questão.

De acordo com os dados pode-se afirmar que dentre os trabalhadores da equipe de APS, a profissão vigia é considerada a mais ansiosa e estressada. Seguida dos AOSD, ASB e ACS. Algumas particularidades da atividade ocupacional dos vigilantes como trabalhar mais de 6 horas diárias e ter que cumprir plantões noturnos potencializam os níveis de estresses dos mesmos. As pessoas que trabalham em período noturno passam por um processo de adaptação do organismo devido à mudança do ritmo circadiano, passado este período o corpo retorna o período de homeostase. Porém muitos trabalhadores apresentam reações diferentes, alguns não retomam o equilíbrio do corpo podendo desenvolver níveis de estresse elevados (Fontes, Neri \& Yassuda, 2010).

Outra característica refere-se ao sono. Pereira-jorge, Espíndola, Varella, Raymundo \& Bernardo (2018) em sua pesquisa afirma que a maioria das pessoas que trabalham em regime de plantões noturno não consegue atingir o ideal de horas de sono por dia ( 8 horas), alegando ter a quantidade e qualidade do sono prejudicadas. Tal situação afeta negativamente nas habilidades físicas e cognitivas do indivíduo, nas relações familiar e social, e na atividade laboral, gerando estresse e ansiedade, impactando negativamente no processo de trabalho como um todo.

A profissão de vigilante é uma categoria profissional ainda pouco estudada no campo da saúde do trabalhador. Os vigilantes são profissionais responsáveis por garantir a segurança e a integridade física dos trabalhadores e visitantes de instituições públicas, como as UBS. Esta característica diretamente ou indiretamente potencializa o estado de estresse e ansiedade do trabalhador, pois permite que o mesmo esteja sujeito a situações de violência, neste caso requer que o profissional esteja em constante estado de alerta e apresente reação rápida a qualquer circunstância que ameace ou viole a segurança (Godinho, Ferreira, Greco, Teixeira \& Teixeira, 2016).

Além do desgaste devido às jornadas de trabalhos com mais de 6 horas diárias, regime de plantão, entre outras, podem predispor alterações fisiológicas, crônicas ou agudas, como o surgimento de DNME. Podendo ainda estar relacionado com mudanças de comportamento, reações psicológicas como a ansiedade e a depressão (Alves, Chor, Faerstein, Lopes \& Werneck, 2004).

Tabela 6- Associação entre os níveis de ansiedade e estresse por outras ocupações.

\begin{tabular}{|c|c|c|c|}
\hline \multirow[t]{2}{*}{ Ansiedade } & \multicolumn{2}{|c|}{ Outra ocupação } & \multirow[t]{2}{*}{ p-valor } \\
\hline & Sim & Não & \\
\hline \multicolumn{4}{|l|}{ Estado } \\
\hline Baixo & $14,3(3)$ & $40,0(20)$ & \multirow[t]{2}{*}{$\mathbf{0 , 0 3 5 * * C}$} \\
\hline Médio & $85,7(8)$ & $60,0(3)$ & \\
\hline \multicolumn{4}{|l|}{ Traço } \\
\hline Baixo & $38,1(8)$ & $38,0(19)$ & \multirow[t]{3}{*}{$0,643^{Q}$} \\
\hline Médio & $52,4(1)$ & $58,0(29)$ & \\
\hline Alto & $9,5(2)$ & $4,0(2)$ & \\
\hline \multicolumn{4}{|l|}{ Estresse no Trabalho } \\
\hline Média \pm Desvio-padrão & $1,9 \pm 0,55$ & $1,8 \pm 0,62$ & $0,173^{\mathrm{K}}$ \\
\hline
\end{tabular}

Q Teste Qui-Quadrado (nível de 95\% de confiança). ${ }^{\mathbf{K}}$ Teste de Kruskal-Wallis (nível de $95 \%$ de confiança).

**Significação estatística.

Fonte: Autores (2020).

Também foram aplicados os testes qui-quadrado e de kruskal-wallis para verificar a associação entre o fato de possuir outra ocupação e os níveis de estado de ansiedade. Pelos resultados, expostos na tabela 6 acima, observa-se uma associação 
significativa entre as variáveis. Nota-se que os trabalhadores que possuem outra ocupação apresentaram nível médio de $\mathrm{AE}$, enquanto àqueles quem não possuem outra ocupação apresentou nível baixo, associações estas estatisticamente significativas (p-valor <0,05). Nas demais variáveis não existem evidências para associações significativas.

Segundo exposto, observa-se que os trabalhadores que possuem outra ocupação, têm níveis maiores de ansiedade e, dentre eles, a profissão de vigilante se mostrou como a categoria que mais possui outra ocupação, seguido das técnicas de enfermagem.

Fernandes, Ribeiro, Santos, Monteiro, Costa \& Soares (2018) afirmam que a prevalência mundial do transtorno de ansiedade (TA) é de 3,6\%, sendo que no continente americano esse transtorno mental alcança maiores proporções e atinge 5,6\% da população, com destaque para o Brasil, onde o TA está presente em 9,3\% da população, possuindo o maior número de casos de ansiedade entre todos os países do mundo.

Somado a isso, casos de depressão, estresse, e de ansiedade têm aumentado a demanda nos consultórios médicos, sendo justificado pela dinâmica capitalista que a sociedade está vivendo, exigindo que muitos profissionais tenham mais de um trabalho, nas quais algumas situações podem favorecer essas estatísticas, inclusive as demandas do ambiente laboral, pois o mercado de trabalho competitivo associado com a angústia do desemprego contribui com que a população se submeta às péssimas condições trabalhistas, estas sendo responsáveis por vários fatores de adoecimento (Leão, Gomes, Ferreira \& Cavalcanti, 2018).

Tabela 7- Associação entre a profissão e DNME.

\begin{tabular}{|c|c|c|c|}
\hline \multirow[t]{2}{*}{ Profissão } & \multicolumn{2}{|c|}{ DNME } & \multirow[t]{2}{*}{ p-valor* } \\
\hline & Sim & Não & \\
\hline AOSD/ASB/ACS & $42,3(11)$ & $53,3(24)$ & \multirow{5}{*}{0,468} \\
\hline $\begin{array}{l}\text { Médico (a)/ Enfermeiro (a) } \\
\text { / Téc. Enfermagem }\end{array}$ & $30,8(8)$ & $24,4(11)$ & \\
\hline Dentista & $3,8(1)$ & $6,7(3)$ & \\
\hline Recepcionista & $0,0(0)$ & $4,4(2)$ & \\
\hline Vigia & $23,1(6)$ & $11,1(5)$ & \\
\hline
\end{tabular}

*Teste Qui-Quadrado (95\% de confiança).

Fonte: Autores (2020).

A tabela 7 evidencia o resultado para a associação entre a profissão e a DNEM. Observe-se que não existem associações significativas entre as variáveis, na qual pode ser justificada pelo tamanho da amostra (71 profissionais), que pode ser considerada pequena. Porém, ao analisar minuciosamente sobre o DNME nota-se que 54,5\% dos vigias apresentam algum distúrbio neuromusculoesquelético com diagnóstico médico. Nota-se também que em todas as profissões presenciou-se a existência de DNME, exceto recepcionista.

Percebe-se que a maior parte da amostra não tem diagnóstico médico de DNME. O que não descarta a possibilidade dos profissionais apresentarem sintomatologia de DNME. Dentre as doenças relacionadas com a saúde do trabalhador, os distúrbios osteomusculares relacionados ao trabalho (DORT) são os mais presentes, gerando desconforto e dores em diferentes graduações de intensidades, além de comprometer vários segmentos corporais (Dosea; Oliveira \& Lima, 2016). Sugere-se investigações mais específicas que abordem esta questão.

Ratificando com este estudo, Ciconato, Cacciar, Haddad, Rossaneis \& Birolim (2016) ao realizar uma pesquisa sobre saúde do trabalhador de uma universidade, afirma que cada função laboral possui característica intrínseca que podem resultar em Lesões por Esforços Repetitivos (LER) e os Distúrbios Osteomusculares Relacionados ao Trabalho (DORT). Sendo os trabalhadores de serviços gerais, inclusive os vigias, os de maior propensão a desenvolver distúrbios 
neuromusculoesqueléticos. Segundo a mesma pesquisa, a categoria profissional que mais compreende trabalhadores readaptados e readequados às suas funções pertence à categoria de serviços gerais.

Exercícios repetitivos, passar muito tempo em posição ortostática, a falta na manutenção da postura ergonomicamente correta, sem cuidado com a ergonomia ao agachar e levantar-se, a sobrecarga musculoesquelética pelo carregamento de peso excessivo e postura incorreta, potencializam os fatores de risco que leva o trabalhador obter uma maior predisposição de doenças ocupacionais, justificado pelo esforço físico extremo e um período curto de recuperação (Leite, Silva \& Merighi, 2007).

A não existência de DNME na profissão de recepcionista deve ser investigada por futuros estudos. Vale ressaltar que para cada unidade básica de saúde tem uma recepcionista, sendo que das 04 UBS analisadas pela pesquisa, duas tem a vaga de recepcionistas ocupadas por Técnicas de Enfermagem, e as mesmas fizeram uso do título de técnica quando foram preencher o quesito profissão do questionário sociodemográfico e ocupacional. Logo, podemos concluir que existe a necessidade de estudos para realizar uma análise por profissão mais detalhada.

Tabela 8- Associação entre possuir outra ocupação e DNME.

\begin{tabular}{|l|c|c|c|}
\hline \multirow{2}{*}{ Outra Ocupação } & \multicolumn{2}{|c|}{ DNME } & p-valor \\
\cline { 2 - 3 } & Sim & Não & \\
\hline Sim & $42,3(11)$ & $22,2(10)$ & 0,074 \\
\hline Não & $57,7(15)$ & $77,8(35)$ & \\
\hline
\end{tabular}

Teste Qui-Quadrado (95\% de confiança).

Fonte: Autores (2020).

Após abordagem estatística, concluiu-se que também não há associação entre possuir outra ocupação e DNEM (pvalor $<0,05$ ) como mostra a Tabela 8. Em contrapartida, os resultados apontam que dos 21 trabalhadores com outra ocupação, 11 possuem DNME.

A hiperutilização do sistema musculoesquelético associado com o pouco espaço de tempo de descanso dentro do espaço laboral potencializa o aparecimento de algumas patologias, podendo gerar incapacidades físicas temporárias e/ou permanentes, e debilitar a saúde mental desses indivíduos (Rocha; Mendes \& Morrone, 2012).

Corroborando com os dados deste estudo, Haeffner et al. (2018) afirma que jornada de trabalho maior que 6 horas diárias pode ter relação com o aparecimento de distúrbios DNME. Associa também, forte correlação com o sexo feminino, uma vez que as mulheres têm mais chances de desenvolver "dupla jornada" em seu dia a dia, entre as tarefas domésticas, como cuidar dos filhos e da casa, somada à rotina diária de trabalho de um ou mais empregos, o que resulta em sobrecarga mental e física, além das diferenças anatomofisiológicas em relação ao homem. Os aspectos de sobrecarga citados podem facilitar o aparecimento de distúrbios musculoesqueléticos e, por conseguinte, levar ao afastamento do trabalho. Vale ressaltar que a amostra desse estudo é característica pela predominância do sexo feminino, correspondendo a 71,8\%.

\section{Conclusão}

Constata-se a predominância do nível médio de ansiedade dentre os profissionais da APS participantes do estudo, sendo os profissionais vigilantes os que demonstraram ter maior estresse e ansiedade. Nota-se que os trabalhadores com mais de uma ocupação apresentam maiores níveis de ansiedade. Sobre o DNME, verifica-se que ele está presente em todas as profissões, exceto recepcionista, e que possui maior predominância também entre os vigilantes. E os resultados apontam que dos 21 trabalhadores com outra ocupação, 11 possuem DNME. 
Diante deste cenário, torna-se necessário desenvolver um olhar mais cuidadoso voltado para a saúde física e mental dos trabalhadores da APS de maneira geral. Mostra-se necessário o fortalecimento do apoio social no trabalho, a importância da ergonomia, dos momentos de interação em equipe e de escuta qualificada, contando com o apoio dos profissionais do NASF e do CEREST (Centros de Referência em Saúde do Trabalhador), além de atividades que possam enriquecer e reorganizar o processo e o fluxo de trabalho, junto com o assessoramento da gestão e dos órgãos da vigilância, e consequentemente evitar demandas exaustivas que possam tornar o ambiente laboral insalubre.

As limitações da pesquisa concentram-se no tamanho pequeno da amostra, na abordagem estritamente quantitativa, na deficiência de completitude de algumas variáveis, o que pode ter reduzido a qualidade do preenchimento dos instrumentos utilizados, e por fim algumas relações de causa e efeito não foram investigadas.

\section{Referências}

Almeida, D. L.M., Ribeiro, L. G., Montibeler, E. E., Domingues Junior, P. L. \& Saldanha, J. A. V. (2017). Estudo sobre os níveis e fatores de estresse profissional: uma análise comparativa entre os setores público e privado. Revista do Congresso de Administração, Sociedade e Inovação. Petrópolis/RJ.

Almeida, D. M. (2015). Satisfação no trabalho e estresse ocupacional na perspectiva dos policiais militares do Estado do Rio Grande do Sul. Dissertação (Mestrado em Administração) -Universidade Federal de Santa Maria, Santa Maria, 148 bp.

Alves, M. G. M., Chor, D., Faerstein, E., Lopes, C. S. \& Werneck, G. L. (2004). Short version of the "job stress scale": a Portuguese-language adaptation. Rev Saúde Pública. 38(2), 164-71.

Brasil. Ministério da Saúde. (2012). Secretaria de Atenção à Saúde. Departamento de Atenção Básica. Política Nacional de Atenção Básica. Ministério da Saúde, Brasília.

Brasil. Ministério da Saúde. (2018). Secretaria de Atenção à Saúde. Secretaria de Vigilância em Saúde. Saúde do trabalhador e da trabalhadora. Brasília: Ministério da Saúde, Cadernos de Atenção Básica, (41), 136p.

Brentini, L. C., Brentini, B. C., Araújo, E. C. S., Aros, A. C. S. P. C. \& Aros, M. S. (2018). Transtorno de ansiedade generalizada no contexto clínico e social no âmbito da saúde mental. Rev. Nucleus. 15(1), abr.

Cardoso, A. C \& Morgado, L. (2019). Trabalho e saúde do trabalhador no contexto atual: ensinamentos da Enquete Europeia sobre Condições de Trabalho. Saúde e Sociedade, 28(1), 169-181.

Carlos, M. A. (2017). Perfil, qualidade de vida, estresse laboral, abuso e dependência de álcool dos trabalhadores da estratégia saúde da família. Dissertação (Mestrado Profissional em Saúde Ambiental e Saúde do Trabalhador) - Universidade Federal de Uberlândia, Instituto de Geografia (PPGAT/UFU). Uberlândia.

Carlotto, M., Câmara, S., Batista, J., \& Schneider, G. (2019). Prevalência de Afastamentos por Transtornos Mentais e do Comportamento Relacionados ao Trabalho em Professores. PSI UNISC, 3(1), 19-32.

Carneiro, C. D. A., Peixoto, S. S., \& Albuquerque, L. C. de. (2021). Occupational stress in professionals of the oral health teams in the family health strategy. Research, Society and Development, 10(4), e17610414065.

Ciconato, A., Cacciar, P., Haddad, M. C. F. L., Rossaneis, M.A. \& Birolim, M. M. (2016). Estado de saúde e perfil ocupacional dos trabalhadores readequados e readaptados de uma universidade pública. Revista de Saúde Pública do Paraná. 17(1), p:49-55.

Dosea, G. S., Oliveira, C. C. C. \& Lima, S. O. (2016). Musculoskeletal symptomatology and quality of life of patients with work-related musculoskeletal disorders. Esc. Anna Nery. 20(4).

Fernandes, B. M. A., Chaves Neto, G., Araújo, P. R. S., Trajano, F. M. P. \& Braga, J. E. F. (2018b). Ansiedade em técnicos de enfermagem da atenção básica. Rev. Electrón. Enferm., (51).

Fernandes, M. A., Ribeiro, H. K. P., Santos, J. D. M., Monteiro, C. F. S., Costa, R. S. \& Soares, R. F. S. (2018). Prevalência de transtornos de ansiedade como causa de afastamento do trabalhador. Revista Brasileira de Enfermagem, 71 (Supl. 5), 2213-2220.

Fontes, A. P., Neri, A. L. \& Yassuda, M. S. (2010). Enfrentamento de estresse no trabalho: relações entre idade, experiência, autoeficácia e agência. Psicol. cienc. prof. 30(3), p:620-33.

Godinho, M. R., Ferreira, A. P., Greco, R. M., Teixeira, L. R. \& Teixeira, M. T. B. (2016). Capacidade para o trabalho e saúde dos vigilantes de uma Universidade pública. Rev. Latino-Am. Enfermagem. 24, p:e2725.

Haeffneri, R., Kalinkeii, L. P., Felliiii, V. E. A., Mantovaniii, M. F., Consonniiv, V. D. \& Sarquisii, L. M. M. (2018). Absenteísmo por distúrbios musculoesqueléticos em trabalhadores do Brasil: milhares de dias de trabalho perdidos. Rev Bras Epidemiol. 21.

Kaipper, M. B. (2008). Avaliação do Inventário de Ansiedade Traço-Estado (IDATE) através da análise de Rasch. Dissertação (Mestrado em Ciências Médicas)-Universidade Federal do Rio Grande do Sul, Porto Alegre. 
Laux, R. C., Hoff, K., Antes, D. L., Cviatkovski, A. \& Corazza, S. T. (2018). Efeito de um Programa de Exercício Físico no Ambiente de Trabalho Sobre a Ansiedade. Rev. Cienc. Trab., 20(62), mai./ago.

Leão, A. M., Gomes, I. P., Ferreira, M. J. M., \& Cavalcanti, L. P. G. (2018). Prevalência e Fatores Associados à Depressão e Ansiedade entre Estudantes Universitários da Área da Saúde de um Grande Centro Urbano do Nordeste do Brasil. Revista Brasileira de Educação Médica, 42(4), 55-65.

Leite, P. C., Silva, A. \& Merighl, M. A. B. (2007). A mulher trabalhadora de Enfermagem e os distúrbios osteomusculares relacionados ao trabalho. Ver. Esc. Enferm. 41(2), p: 287-91.

Morin, E., Tonelli, M. J. \& Pliopas, A. L. V. (2007). O trabalho e seus sentidos. Psicol Soc. 19, 47-56.

Oliveira, M. M. C. (2007). Presença e extensão dos atributos da Atenção Primária à Saúde entre os serviços de Atenção Primária em Porto Alegre: uma análise agregada. Dissertação - Universidade Federal do Rio Grande do Sul, Porto Alegre.

Pereira-jorge, I. M., Espíndola, T. K., Varella, P. B.,Raymundo, T. M. \& Bernardo, L. D. (2018). Identificação do estresse em trabalhadores do período noturno Identification of stress among night shift workers. Rev. Fac. Med. 66(3), 327-33.

Pie, A. C. S., Fernandes, R. C. P., Carvalho, F. M., \& Porto, L. A. (2020). Fatores associados ao presenteísmo em trabalhadores da indústria. Revista Brasileira de Saúde Ocupacional, 45, e13. Epub April 03, 2020.

Ribeiro, R. P., Marziale, M. H. P., Martins, J. T., Ribeiro, P. H. V., Robazzi, M. L. C. C. \& Dalmas, J. C. (2015). Prevalência da Síndrome Metabólica em trabalhadores de enfermagem e sua associação com estresse ocupacional, ansiedade e depressão. Revista Latino-Americana de Enfermagem, 23 (3), 435440. Epub em 03 de julho de 2015.

Rocha, S. R.A., Mendes, A. M. \& Morrone, C. F. (2012). Sofrimento, Distúrbios osteomoleculares e Depressão no contesto de trabalho. Estudos e Pesquisa em Psicologia. 12(2), 379-394.

Souza, M. A. \& Stancato, K. (2010). Avaliação da Qualidade de Vida de Profissionais de Saúde em Campinas. Rev Adm Saúde. 12(49), 154-162. 